\title{
Interference Characteristics in Power-Controlled Cognitive Radio Networks
}

\author{
Amir Ghasemi \\ Communications Research Centre Canada \\ 3701 Carling Ave., Ottawa, ON, Canada \\ Tel: +1 (613) 949-5592 \\ Email: aghasemi@crc.gc.ca
}

\begin{abstract}
Dynamic spectrum access using cognitive radios has been proposed as an approach to meet the increasing demand for radio spectrum. Under this novel access paradigm unlicensed cognitive radios are allowed to utilize the white spaces across licensed spectrum on a non-interfering basis. Realization of this concept therefore requires a model translating the regulatory constraint on aggregate interference to system design parameters.

Thus far, a few models of aggregate interference have been proposed however, these mainly examine the distribution of interference without explicitly modelling transmit power control. In this paper a statistical model of interference aggregation in power-controlled cognitive radio networks is developed. In particular, distribution of the aggregate interference is characterized in terms of parameters such as density of cognitive radios and access points as well as the underlying propagation environment. The model is further extended to account for the effect of correlated shadowing on the distribution of aggregate interference.
\end{abstract}

\section{INTRODUCTION}

The increasing demand for radio spectrum and the relatively inefficient usage of the licensed frequency bands have led to a debate as to how the spectrum access should be regulated. In particular, regulatory bodies are investigating a new access paradigm where secondary (unlicensed) systems are allowed to dynamically access the unused primary (licensed) bands, commonly referred to as the white spaces.

Perhaps the most crucial task in the implementation of this revolutionary access paradigm is how to prevent secondary users from adversely interfering with the incumbent primary systems. This requires the secondary systems to have a level of awareness about their impact on the primary systems through techniques such as spectrum sensing or geo-location [1].Such awareness is one of the main functionalities envisaged for cognitive radios [2]. Consequently, cognitive radio (CR) is increasingly perceived as the main technology enabling dynamic spectrum access (DSA) [3].

In essence, both spectrum sensing and geo-location techniques protect the incumbents by establishing a guard (inhibition) zone around the primary system where cognitive radios are not allowed to transmit [4]. Size of this guard zone controls the level of total interference experienced by primary system and therefore should be set such that spatial frequency reuse is maximized while adequately protecting the primary system. Selection of the optimum guard zone is however challenged by the uncertainty in aggregate interference which in turn results from variations in the number, location, and transmitted power of individual cognitive radios as well as random radio propagation effects.

Transmit power control is a conventional method of controlling the interference. While the impact of power control in cellular systems has been widely studied, its effect on interference generated by CR networks and thereby on parameters such as size of the primary guard zone have not been quantified yet. In this paper we develop a statistical model of interference aggregation in power-controlled cognitive wireless networks by explicitly taking into account the random variations in number and location of the cognitive radios as well as propagation characteristics [5]. The derivations highlight potential benefits of power control in the context of DSA as well as the interplay among various system and propagation parameters hence forming a preliminary basis for development of more comprehensive models of interference in CR networks.

The remainder of this paper is organized as follows. Related past research and system model are summarized in Sections II and III, respectively. Distribution of the aggregate interference in a power-controlled cognitive radio network is characterized in Section IV. Impact of various system parameters on interference is investigated in Section $\mathrm{V}$ through numerical analysis. In Section VI the results are extended to the case with correlated shadowing. Finally, some concluding remarks are presented in Section VII.

\section{RELATED WORK}

Characteristics of aggregate interference in a Poisson field of interferers were first studied by Sousa and Silvester in [6] where the interference was shown to follow a stable distribution with infinite variance. This result was extended to Rayleigh fading channels in [7]. It was later shown in [8] through simulations that inclusion of a large guard (inhibition) zone around the receiver to suppress the nearby interferers would result in approximately Gaussian aggregate interference.

Several recent studies have attempted to model the characteristics of aggregate interference in cognitive radio networks. In [5] we studied the effect of detection errors induced by channel fading on distribution of interference in spectrumsensing CR networks. The impact of power control on the aggregate interference of cognitive wireless networks was first considered in [9]. The authors however, did not take the 
effect of random user locations into account and as such, modelled the aggregate interference as a deterministic value through its average. In other words, authors did not study the distribution of interference around its average resulting from the randomness in user locations and propagation channels.

More recently, distribution of aggregate interference in a CR network with a guard zone around the primary receiver was studied in [10]. Authors however, did not study power control and relied on numerical methods to obtain the probability of interference crossing a ceratin threshold.

\section{SySTEM MODEL}

\section{A. Channel Model}

We model the propagation power loss between a secondary user and an access point (AP) at a distance $r$ by $g(r) X$ where $g(r)$ is the distance-dependent path-loss and $X \in \mathbb{R}^{+}$is a unit-mean random variable representing the frequency-flat shadowing or fading effects. We assume $X$ to be independent and identically distributed (i.i.d.) across different users and also independent of the distance $r$, with $f_{X}($.$) and F_{X}($. denoting its probability density function (PDF) and cumulative distribution function (CDF), respectively. In a similar manner, propagation power loss between a secondary user and a primary receiver is modelled as $g(r) Y$ with $Y$ being a distanceindependent unit-mean random variable. Same as with $X, Y$ is assumed to be i.i.d. across different users.

In what follows, for any given user, $X$ and $Y$ are assumed to be independent of each other and identically distributed however, the independence assumption will be relaxed in Section VI where we consider correlated shadowing. Extension to the general case with non-identical distributions for $X$ and $Y$ is straightforward.

The path-loss in turn is usually modelled as $g(r)=\mathrm{Cr}^{-\alpha}$ where $\alpha>2$ is the path-loss exponent and $C$ is a constant.

\section{B. Network Model}

We consider a single primary receiver, located at the origin, within a large secondary cognitive radio network. We also assume a guard zone of radius $R_{g}$ around the primary receiver inside which no secondary user is allowed to transmit. Such guard zone may be established through either spectrum sensing [5] or a geolocation-based approach where users compare their location against a database of active primary users [1].

The secondary transmitters and access points are assumed to be distributed over $\mathbb{R}^{2}$ according to two independent homogeneous Poisson point processes with intensities of $\lambda$ and $\lambda_{a p}$, respectively. Such a model, for instance, has been recently applied to represent a network of randomly distributed femtocells and their users [11].

Each secondary user is assumed to be associated to and power-controlled by its nearest AP. A conventional approach to modelling the power control is for the users to maintain a fixed received power level, denoted by $p_{0}$, at their corresponding AP. Using this approach, the transmitted power of the $i$ th
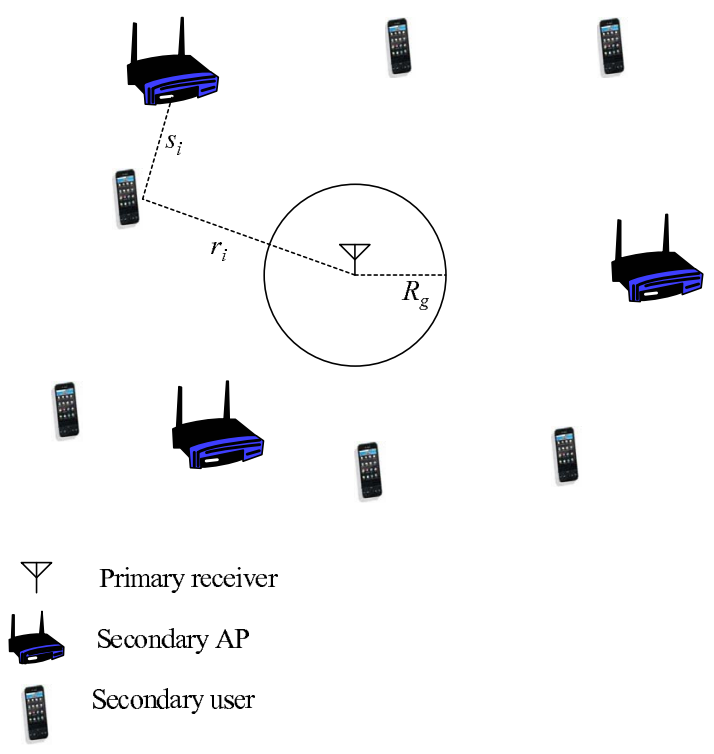

Fig. 1: Network model with a guard zone around the primary receiver

secondary user, located at a distance $s_{i}$ from its nearest access point, is given by,

$$
p_{i}=\frac{p_{0}}{g\left(s_{i}\right) y_{i}}
$$

where $y_{i}$ is the realization of $Y$ at the $i$ th user. From the characteristics of Poisson point processes, the distance to nearest AP, $S$, has the following distribution,

$$
f_{S}(s)=2 \pi \lambda_{a p} s e^{-\lambda_{a p} \pi s^{2}}, \quad s \geq 0
$$

In what follows cognitive radios are assumed to transmit independently of each other. This may for instance represent a spread spectrum system where intra-system interference is managed through spreading. Alternatively, the secondary network may employ a spatial reuse protocol such as carrier sensing multiple access (CSMA) to control its intra-system interference. This would limit the number of simultaneously transmitting secondary users and therefore would further reduce the interference caused to primary system. In this paper however, we focus on interference mitigation through power control only and do not consider the effect of such medium access protocols.

\section{Interference Protection}

We assume that the primary receiver, when active, is capable of tolerating a maximum interference power of $I_{t h}$. Interference levels higher than this threshold are considered harmful and should be avoided. Therefore, the regulatory constraint on the aggregate interference of cognitive radios, $I$, may be expressed as,

$$
P\left[I>I_{t h}\right] \leq \beta
$$

where $\beta \ll 1$ is the maximum permissible probability of harmful interference and should be supplied either by the regulator or the primary operator. Throughout the paper we will use the 
term interference probability to refer to $P\left[I>I_{t h}\right]$.

Understanding the constraint specified by (3) and its implications in terms of system and protocol design are pivotal to the successful deployment of cognitive wireless networks in licensed bands. In particular, this regulatory constraint needs to be translated into appropriate engineering guidelines for the system designer. This will be achieved by characterizing the distribution of the aggregate interference in terms of parameters such as the primary guard zone radius, secondary users' and access points' spatial density, and finally the propagation characteristics of the environment.

\section{Distribution of the Aggregate Interference}

Let $\Pi$ be a point process consisting of the location of all secondary transmitters in $\mathbb{R}^{2} \backslash b\left(o, R_{g}\right)$, where $\backslash$ and $b\left(o, R_{g}\right)$ denote the set exclusion operator and a disk of radius $R_{g}$ centered at the primary receiver, respectively. Also, without loss of generality, let $p_{0}=1$. For all other cases, the distribution of the aggregate interference may be properly scaled as needed. The normalized aggregate interference experienced by the primary receiver then may be written as ${ }^{1}$,

$$
\begin{aligned}
I & =\sum_{i \in \Pi} \frac{x_{i}}{y_{i}}\left(\frac{r_{i}}{s_{i}}\right)^{-\alpha} \\
& =\sum_{i \in \Pi} z_{i}\left(\frac{r_{i}}{s_{i}}\right)^{-\alpha}
\end{aligned}
$$

where $z_{i}=x_{i} / y_{i}$ is the realization of the new random variable $Z=X / Y$ at the $i$ th user.

Using an approach similar to [6] it is relatively straightforward to show that the characteristic function of $I$, denoted by $\Psi_{I}(\omega)$, may be written as,

$$
\begin{aligned}
\Psi_{I}(\omega) & =E\left[e^{j \omega I}\right] \\
& =\exp \left(2 \pi \lambda E_{S, Z}\left[\int_{R_{g}}^{\infty}\left(\exp \left(j \omega z(r / s)^{-\alpha}\right)-1\right) r d r\right]\right)
\end{aligned}
$$

where $E_{S, Z}[$.$] denotes expectation with respect to random$ variables $S$ and $Z$. This expression generally does not admit closed-form solutions and has to be numerically inverted to compute the exact PDF of the aggregate interference (see e.g., [12]). However, this approach is not pursued here as it is computationally intensive and offers little insight into the interplay of aggregate interference and various system parameters. On the other hand (5) may be used to derive exact and possibly closed-form expressions for the cumulants of the aggregate interference. The $n$th cumulant of $I$ may be obtained from (5) as,

$$
\kappa_{n}=\frac{1}{j^{n}}\left[\frac{\partial^{n} \ln \Psi_{I}(\omega)}{\partial \omega^{n}}\right]_{\omega=0}
$$

\footnotetext{
${ }^{1}$ We assume a frequency division duplex (FDD) system where APs transmit in a different band and therefore limit our analysis to interference generated by secondary users.
}

$$
\begin{aligned}
& =2 \pi \lambda E_{S, Z}\left[\int_{R_{g}}^{\infty} z^{n} r^{-n \alpha} s^{n \alpha} r d r\right] \\
& =2 \pi \lambda \int_{0}^{\infty} \int_{0}^{\infty} \int_{R_{g}}^{\infty} z^{n} r^{1-n \alpha} s^{n \alpha} f_{Z}(z) f_{S}(s) d r d z d s \\
& =2 \pi \lambda E_{Z}\left[z^{n}\right] \int_{0}^{\infty} \int_{R_{g}}^{\infty} r^{1-n \alpha} s^{n \alpha} f_{S}(s) d r d s \\
& =\frac{2 \pi \lambda E_{Z}\left[z^{n}\right]}{(n \alpha-2) R_{g}^{n \alpha-2}} \int_{0}^{\infty} s^{n \alpha} f_{S}(s) d s
\end{aligned}
$$

Substituting for $f_{S}(s)$ from (2) and taking the expectation over $S$, (6) simplifies to,

$$
\begin{aligned}
\kappa_{n} & =\frac{2 \pi \lambda E_{Z}\left[z^{n}\right]}{(n \alpha-2) R_{g}^{n \alpha-2}} \int_{0}^{\infty} 2 \pi \lambda_{a p} s e^{-\lambda_{a p} \pi s^{2}} s^{n \alpha} d s \\
& =\frac{2 \pi \lambda E_{Z}\left[z^{n}\right] \Gamma\left(\frac{n \alpha}{2}+1\right)}{(n \alpha-2) R_{g}^{n \alpha-2}\left(\pi \lambda_{a p}\right)^{n \alpha / 2}}
\end{aligned}
$$

where $\Gamma($.$) is the Gamma function. Therefore, for \alpha>2$, $\kappa_{n}$ exists as long as $E_{Z}\left[z^{n}\right]$ is finite. In this case, having the exact cumulants (and hence moments) of $I$ at our disposal, we may employ various methods to approximate the distribution of the aggregate interference. In this paper we employ the shifted log-normal approximation which has been shown to offer good accuracy using only the first three cumulants [13]. The interested reader is referred to [14] and [15], among other references, for additional techniques which may offer improved accuracy at an increased complexity.

\section{A. AWGN Channel}

First let us consider the simple case of non-fading AWGN channel where $Z$ is deterministic and equal to unity for all users, i.e. $f_{Z}(z)=\delta(z-1)$. The cumulants of $I$ for this scenario may be simplified as,

$$
\kappa_{n}=\frac{2 \pi \lambda \Gamma\left(\frac{n \alpha}{2}+1\right)}{(n \alpha-2) R_{g}^{n \alpha-2}\left(\pi \lambda_{a p}\right)^{n \alpha / 2}}
$$

\section{B. Log-normal Shadowing}

Empirical measurements suggest that the medium-scale variations of received power, when represented in $\mathrm{dB}$ units, follow a normal distribution. In this case, $X$ and $Y$ may be modelled as $e^{\sigma W_{1}}$ and $e^{\sigma W_{2}}$, respectively, where $W_{1}$ and $W_{2}$ are zero-mean, unit-variance Gaussian random variables. Lognormal shadowing is usually characterized in terms of its dBspread which is related to $\sigma$ by $\sigma=0.1 \log (10) \sigma_{d B}$. The $\mathrm{dB}$-spread typically ranges from 4 to $12 \mathrm{~dB}$ as indicated by empirical measurements [16].

With $X$ and $Y$ being independent log-normal random variables as defined above, $Z=e^{\sigma W}$ where $W=W 1-W 2$ is a zero-mean Gaussian r.v. with a variance of $2 \sigma^{2}$. Then it is easy to show,

$$
\begin{aligned}
E_{Z}\left[z^{n}\right] & =\frac{1}{\sqrt{2 \pi}} \int_{-\infty}^{\infty} e^{n \sqrt{2} \sigma z-\frac{z^{2}}{2}} d z \\
& =e^{n^{2} \sigma^{2}}
\end{aligned}
$$




\section{Approximation of Interference Probability}

By virtue of the central limit theorem (CLT) one may be tempted to approximate the distribution of $I$ with a Gaussian PDF. However, inspection of the cumulants shows that the PDF of $I$ is positively skewed and thus deviates from normality. This issue becomes more pronounced with a smaller guard zone around the primary receiver $\left(R_{g} \downarrow\right)$ since there will be a higher chance of having strong interferers located close to the primary receiver which in turn results in a heavier right tail for the distribution of $I$.

For the case without power control, in [5] we proposed to approximate $I$ with a three-parameter shifted log-normal (SLN) random variable $Z$ with the following PDF,

$$
f_{Z}(z)=\frac{1}{\sigma(z-c) \sqrt{2 \pi}} e^{-(\log (z-c)-\mu)^{2} / 2 \sigma^{2}}, \quad z>c
$$

where $c$ is the shift parameter. This enables us to match the skewness of $Z$, in addition to its mean and variance, to those of $I$. Applying the method of moments, $\mu, \sigma^{2}$ and $c$ may be obtained in terms of the first three cumulants of $I$ as shown below (see e.g., [17]),

$$
\begin{aligned}
\sigma^{2} & =\log \left(1+\left[\sqrt[3]{\frac{\kappa_{3}}{2}+\sqrt{\frac{\kappa_{3}^{2}}{4}+1}}+\sqrt[3]{\frac{\kappa_{3}}{2}-\sqrt{\frac{\kappa_{3}^{2}}{4}+1}}\right]^{2}\right) \\
\mu & =\frac{1}{2}\left[\log \left(\frac{\kappa_{2}}{e^{\sigma^{2}}-1}\right)-\sigma^{2}\right] \\
c & =\kappa_{1}-e^{\mu+\frac{\sigma^{2}}{2}}
\end{aligned}
$$

Consequently,

$$
P\left[I>I_{t h}\right] \simeq Q\left(\frac{\log \left(I_{t h}-c\right)-\mu}{\sigma}\right)
$$

with $\mu, \sigma$ and $c$ obtained from (10)-(12). It was observed in [5] that the SLN approximation provides a good fit in the tail region of the PDF.

For the power-controlled CR network described in this paper, one may employ any cumulant-based technique to approximate the PDF of $I$. However, in order to illustrate the application of our results we have used the above SLN approximation. As seen in Fig. 2, the SLN distribution provides a better fit in the tail region of the PDF compared to lognormal and Gamma, two other commonly used distributions.

Using the SLN approximation, in Fig. 3, $\lambda_{a p}$ required to maintain the interference probability at 0.01 is plotted as a function of $R_{g}$. We observe that on a log-log plot $\lambda_{a p}$ is a linearly decreasing function of $R_{g}$. Moreover, a change in the density of cognitive radios, $\lambda$, results only in a shift of $\lambda_{a p}$ which is equivalent to a scaling factor on the linear scale. Therefore, for a given $\lambda$, as more and more access points are deployed within the CR network, the guard zone around the primary receiver may be reduced thereby enabling higher spatial reuse of spectrum.

Fig. 4 shows the required $\lambda_{a p}$ as a function of $\lambda$. Comparing

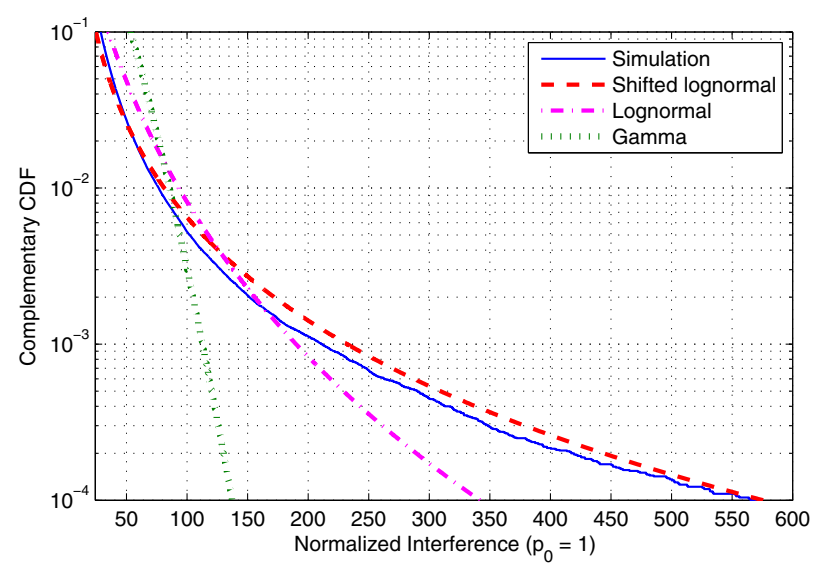

Fig. 2: Complementary CDF of normalized aggregate interference under log-normal shadowing $\left(\sigma_{d B}=6\right), \lambda=0.01$, $\lambda_{a p}=0.001, \alpha=4, R_{g}=50$.

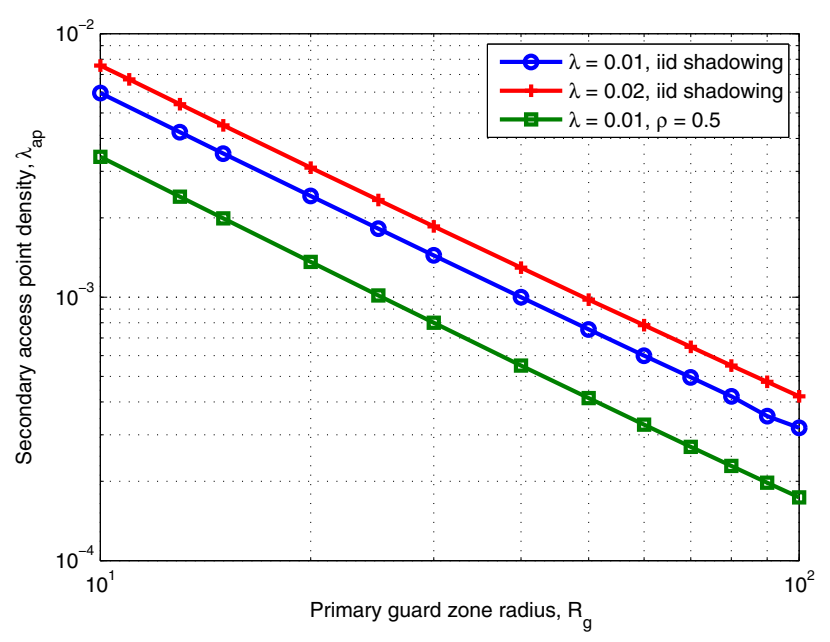

Fig. 3: Secondary AP density required to maintain the interference probability at 0.01 as a function of guard zone radius (log-normal shadowing with $\sigma_{d B}=6, \alpha=4, I_{t h}=100$ ).

the plots for various values of $R_{g}$ and $\alpha$, we observe that a smaller path-loss exponent results in a sharper increase in the required AP density as user density grows. Furthermore, $R_{g}$ may be traded off with $\lambda_{a p}$ specially at higher user densities where the required AP density may become prohibitively large.

\section{CORRELATED FADING}

Up to this point, the derivations were based on the assumption that $X$ and $Y$ vary independently. Consider a scenario where the $i$ th cognitive radio's distance to its corresponding access point and the primary receiver is in the same order. Then if $y_{i}$ is very small compared to $x_{i}$, this user will significantly contribute to the aggregate interference caused to the primary receiver. We note, however, that in practice there is usually a degree of correlation between $X$ and $Y$ due to similar local propagation effects at the cognitive transmitter. 


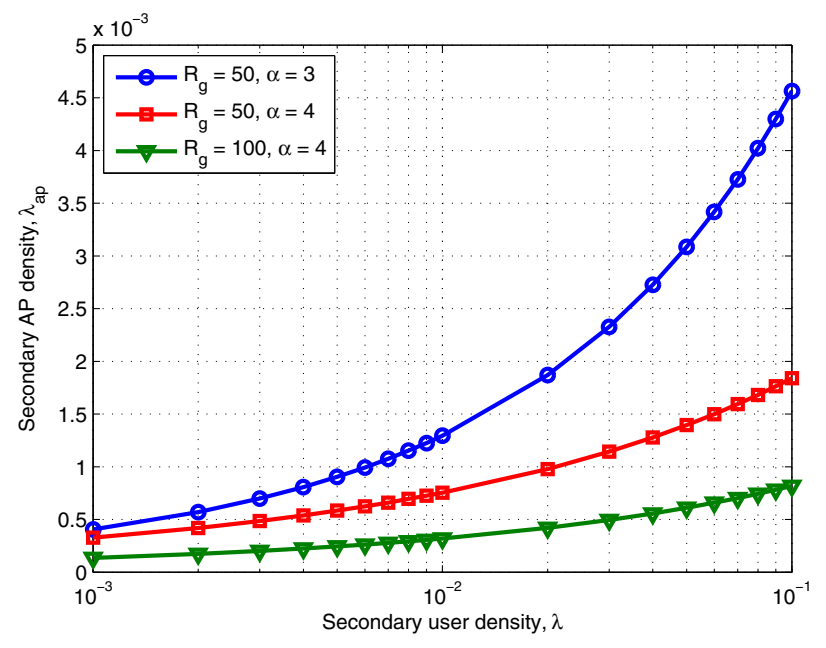

Fig. 4: Secondary AP density required to maintain the interference probability at 0.01 as a function of user density (lognormal shadowing with $\sigma_{d B}=6, I_{t h}=100$ ).

This correlation in turn would reduce the probability of such events. In this section we examine the impact of shadowing correlation on the distribution of aggregate interference.

The correlation between log-normally distributed $X$ and $Y$ may be considered via a joint Gaussian pdf for $U_{0}=\log X$ and $U_{1}=\log Y$. Equivalently, one may model $U_{0}$ and $U_{1}$ as follows [18],

$$
U_{i}=\sqrt{\rho} U+\sqrt{1-\rho} V_{i}, \quad i=0,1
$$

where $U, V_{0}$ and $V_{1}$ are independent zero-mean Gaussian random variables with equal variance of $\sigma^{2}$ and $\rho$ is the correlation coefficient between $U_{0}$ and $U_{1}$. It can be seen that $X$ and $Y$ become equal if $\rho$ is set to 1 and they would be independent log-normal variables if $\rho=0$. Under the model defined by (14) $X / Y$ is given by,

$$
Z=\frac{X}{Y}=e^{\sqrt{1-\rho}\left(V_{0}-V_{1}\right)}
$$

It can be seen that as in the i.i.d. case, $X / Y$ is log-normally distributed. However, $\log X / Y$ has a variance of $2(1-\rho) \sigma^{2}$ instead of $2 \sigma^{2}$. Thus, the previous results apply here as well if the $\mathrm{dB}$ spread of the log-normal shadowing is scaled by $\sqrt{1-\rho}$.

Fig. 3 illustrates the impact of correlated shadowing on the required AP density. It is observed that a correlation factor of 0.5 significantly scales down the required $\lambda_{a p}$.

\section{CONCLUding REMARKS}

Previous research has mostly examined the statistics of interference in cognitive radio networks without considering the effect of power control. In this paper we developed a statistical model of interference aggregation in power-controlled cognitive radio networks by taking into account the random variations in the number, location and transmitted power of the cognitive radios. The results were used to approximate the probability of harmfully interfering with a licensed receiver in terms of the density of cognitive users and access points, the primary guard zone's radius and finally the propagation characteristics.

The presented results provide a means of investigating the interplay among various system and propagation parameters and form a preliminary basis upon which more comprehensive models of interference aggregation, as needed for spectrum sharing in DSA systems, may be built.

\section{REFERENCES}

[1] Federal Communications Commission, "Second Report and Order and Memoandum Opinion and Order, in the matter of unlicensed operation in the TV broadcast bands (ET Docket No. 04-186) and additional spectrum for unlicensed devices below $900 \mathrm{MHz}$ and in the $3 \mathrm{GHz}$ band (ET Docket No. 02-380), FCC 08-260," November 2008.

[2] J. Mitola et al., "Cognitive radio: Making software radios more personal," IEEE Personal Communications, vol. 6, no. 4, pp. 13-18, August 1999.

[3] Federal Communications Commission, "Notice of Proposed Rulemaking, in the matter of facilitating opportunities for flexible, efficient and reliable spectrum use employing cognitive radio technologies (ET Docket No. 03-108) and authorization and use of software defined radios (ET Docket No. 00-47), FCC 03-322,” December 2003.

[4] S. J. Shellhammer, "A comparison of geo-location and spectrum sensing in cognitive radio," in Proc. 18th International Conference on Computer Communications and Networks (ICCCN'09), August 2009.

[5] A. Ghasemi and E. S. Sousa, "Interference aggregation in spectrumsensing cognitive wireless networks," IEEE Journal of Selected Topics in Signal Processing, vol. 2, no. 1, pp. 41-56, February 2008.

[6] E. S. Sousa and J. A. Silvester, "Optimum transmission ranges in a direct-sequence spread-spectrum multihop packet radio network," vol. 8, no. 5, pp. 762-771, June 1990.

[7] M. Souryal, B. Vojcic, and R. Pickholtz, "Ad hoc, multihop CDMA networks with route diversity in a Rayleigh fading channel," in Proc. IEEE Military Communications Conference (MILCOM'01), pp. 10031007, October 2001.

[8] A. Hasan and J. G. Andrews, "The guard zone in wireless ad hoc networks," submitted to IEEE Transactions on Wireless Communications, available at: http://www.ece.utexas.edu/ ahasan/research.htm.

[9] R. Tandra and A. Sahai, "Power scaling for cognitive radio," in Proc. International Conference on Wireless Networks, Communications and Mobile Computing, pp. 250-255, June 2005.

[10] X. Hong, C. Wang and J. Thompson, "Interference modeling of cognitive radio networks," in Proc. IEEE Vehicular Technology Conference (VTC'08-Spring), pp. 1851-1855, May 2008.

[11] V. Chandrasekhar and J. G. Andrews, "'Uplink capacity and interference avoidance for two-tier femtocell networks," IEEE Transactions on Wireless Communications, vol. 8, no. 7, pp. 3498-3509, July 2009.

[12] J. Abate and W. Whitt, "The Fourier-series method for inverting transmissions of probability distributions," Queueing Systems, vol. 10, pp. 5-88, 1992.

[13] A. Ghasemi, "Statistical characterization of interference in cognitive radio networks," in Proc. IEEE international symposium on Personal, Indoor and Mobile Radio Communications (PIMRC'08), September 2008.

[14] W. P. Elderton and N. L. Johnson, System of Frequency Curves. Cambridge: Cambridge University Press, 1969.

[15] J. L. Jensen, Saddlepoint Approximations. Oxford: Oxford University Press, 1995.

[16] A. J. Goldsmith, Wireless Communications. Cambridge University Press, 2005.

[17] K. L. Q. Read, "A lognormal approximation for the collector's problem," The American Statistician, vol. 52, no. 2, pp. 175-180, May 1998.

[18] A. J. Viterbi, A. M. Viterbi and E. Zehavi, "Other-cell interference in cellular power-controlled CDMA," IEEE Transactions on Communications, vol. 42, no. 2/3/4, pp. 1501-1504, February/March/April 1994. 